\title{
Characterization and Imaging of Lipid-Shelled Microbubbles for Ultrasound-Triggered Release of Xenon
}

\author{
Himanshu Shekhar ${ }^{1}$ (D) - Arunkumar Palaniappan ${ }^{1} \cdot$ Tao Peng $^{2} \cdot$ Maxime Lafond $^{1} \cdot$ Melanie R. Moody $^{2}$. \\ Kevin J. Haworth ${ }^{1,3}$. Shaoling Huang ${ }^{2}$. David D. McPherson ${ }^{2}$. Christy K. Holland ${ }^{1,3}$
}

Published online: 24 April 2019

(C) The American Society for Experimental NeuroTherapeutics, Inc. 2019

\begin{abstract}
Xenon (Xe) is a bioactive gas capable of reducing and stabilizing neurologic injury in stroke. The goal of this work was to develop lipid-shelled microbubbles for xenon loading and ultrasound-triggered release. Microbubbles loaded with either xenon (Xe-MB) or xenon and octafluoropropane (Xe-OFP-MB) $(9: 1 v / v)$ were synthesized by high-shear mixing. The size distribution and the frequency-dependent attenuation coefficient of Xe-MB and Xe-OFP-MB were measured using a Coulter counter and a broadband acoustic attenuation spectroscopy system, respectively. The Xe dose was evaluated using gas chromatography/mass spectrometry. The total Xe doses in Xe-MB and Xe-OFP-MB were $113.1 \pm 13.5$ and $145.6 \pm 25.5 \mu \mathrm{l}$ per mg of lipid, respectively. Co-encapsulation of OFP increased the total xenon dose, attenuation coefficient, microbubble stability (in an undersaturated solution), and shelf life of the agent. Triggered release of gas payload was demonstrated with 6-MHz duplex Doppler and 220$\mathrm{kHz}$ pulsed ultrasound. These results constitute the first step toward the use of lipid-shelled microbubbles for applications such as neuroprotection in stroke.
\end{abstract}

Key Words Bioactive gas delivery $\cdot$ xenon delivery $\cdot$ lipid-shelled microbubbles $\cdot$ ultrasound $\cdot$ cytoprotection.

\section{Introduction}

Stroke is the fifth leading cause of death in the USA and costs over $\$ 34$ billion annually in healthcare expenses and lost productivity [1]. Ischemic stroke, intracerebral hemorrhage, and subarachnoid hemorrhage comprise approximately $87 \%$, $10 \%$, and $3 \%$, of all stroke cases, respectively [1]. Despite advances in stroke treatment, therapies are needed to arrest neuronal damage caused by excitotoxicity, inflammation, oxidative stress, and edema [2-5]. Excitotoxicity caused by extracellular glutamate accumulation is an important mechanism of neuronal injury [6]. Pharmacological approaches based on

Himanshu Shekhar

h.shekhar.uc@gmail.com

1 Division of Cardiovascular Health and Disease, Department of Internal Medicine, University of Cincinnati, Cincinnati, Ohio, USA

2 Division of Cardiovascular Medicine, Department of Internal Medicine, University of Texas Health Science Center at Houston, Houston, Texas, USA

3 Department of Biomedical Engineering, University of Cincinnati, Cincinnati, Ohio, USA inhibition of glutamate receptors are under development for neuroprotection [7-10].

Xenon, a neuroprotectant gas, acts by mechanisms such as the inhibition of glutamatergic effects of $N$-methyl-D-aspartate (NMDA) receptors along with the promotion and transcription of prosurvival genes $[10,11]$. Additionally, Xe alleviates ischemic injury by altering the cellular pathways involved in neuronal ischemic tolerance and protecting against oxygen and glucose deprivation [11-15]. The small size and low blood-gas partition coefficient of Xe enables this gas to cross the blood-brain barrier and to reach target tissues rapidly [11].

The neuroprotective action of $\mathrm{Xe}$ is dose dependent [11]. Inhalation of subanesthetic concentrations of Xe (50-70\% by volume) promotes neuroprotection in murine models [11]. However, $\mathrm{Xe}$ is a rare gas, which makes its administration by inhalation expensive and is therefore used with mechanical ventilation [16]. Localized delivery could enhance the bioavailability of Xe during ischemic stroke therapy [17, 18]. Our group has reported the delivery of bioactive gases such as Xe [17-19] and nitric oxide [20,21] using echogenic liposomes (ELIP). The neuroprotective effects of Xe-loaded echogenic liposomes (Xe-ELIP) have been demonstrated in rat models of ischemic stroke, subarachnoid hemorrhage, 
and depression by both intravenous and intra-arterial administration of the agent [17-19, 22]. Echogenic liposomes consist of a lipid bilayer and an aqueous core with up to $10 \%$ of the total volume composed of gas [23]. Up to $34 \%$ of the XeELIP payload exists in dissolved form in the lipid bilayer [17], which enables a biphasic release profile [19]. Shelled microbubbles have been investigated for the delivery of bioactive gases such as oxygen, nitric oxide, and hydrogen sulfide [24-29]. The volume of microbubbles is almost entirely composed of gas, which enables ultrasound-triggered release and enhancement of payload delivery. Lipid-shelled microbubbles are stabilized by the co-encapsulation of octafluropropane (OFP) [24, 30]. OFP is characterized by low aqueous solubility, low diffusivity, and biological inertness [31, 32] and is used in the FDA-approved ultrasound contrast agent Definity® (Lantheus Medical Imaging, North Billerica, MA, USA) [33]. However, the effect of coencapsulating OFP on Xe loading and stability of lipidshelled microbubbles has not been investigated previously.

The goal of this study was to evaluate the Xe loading, acoustic response, and stability of microbubbles synthesized with either xenon alone (Xe-MB) or with $90 \%$ Xe and $10 \%$ OFP by volume (Xe-OFP-MB). The size distribution of these agents was measured along with the acoustic attenuation coefficient. Xenon loading was quantified using gas chromatography coupled with mass spectrometry (GC/MS). Finally, the feasibility of releasing the Xe payload of microbubbles was evaluated using 6-MHz duplex Doppler and 220-kHz pulsed ultrasound.

\section{Methods}

\section{Microbubble Materials}

1,2-Distearoyl-sn-glycero-3-phosphocholine (DSPC) and 1,2distearoyl-sn-glycero-3-phos phoethanolamineN-[methoxy(polyethylene glycol)-2000] (18:0 PEG2000 PE) were purchased from Avanti Polar Lipids (Alabaster, AL, USA). Xenon (99.999\%) and OFP gas (99.9\%) were purchased from Concorde Specialty Gases Inc. (Eatontown, NJ, USA) and Advanced Specialty Gases (Reno, NV, USA), respectively. Bovine serum albumin (BSA), phosphate buffered saline (PBS) tablets, glycerol, and propylene glycol were purchased from Sigma Aldrich (St. Louis, MO, USA).

\section{Preparation of Lipid-Shelled Microbubbles Containing Xenon}

To prepare Xe-loaded microbubbles, DSPC and 18:0 PEG2000 PE were added in a round-bottom flask in a molar ratio of 9:1. The solvents were evaporated, and a thin film was formed on the inner surface of the flask using a rotary evaporator (N-1001, Eyela, Bohemia, NY). The flask was connected to a lyophilizer (Labconco FreeZone 2.5, Labconco, Kansas City, MO, USA) overnight to remove residual solvents completely. On the following day, the lipids were rehydrated using a buffer solution (PBS:propylene glycol:glycerol $=16: 3: 1 \mathrm{v} / \mathrm{v}$ ) prewarmed to $60{ }^{\circ} \mathrm{C}$ using a water bath, to obtain a final lipid concentration of $1 \mathrm{mg} / \mathrm{ml}$. After rehydration (indicated by the removal of the thin film from the wall of the flask), the flask was rotated for $3 \mathrm{~min}$ at $60^{\circ} \mathrm{C}$ and subsequently for $30 \mathrm{~min}$ at room temperature. Thereafter, the reconstituted lipids were sonicated (Branson 3510, Branson Ultrasonics, Danbury, CT, USA) for $15 \mathrm{~min}$, transferred to a 20-ml scintillation glass vial, and sonicated for another $15 \mathrm{~min}$ to obtain a clear lipid dispersion. Aliquots $(1.4 \mathrm{ml})$ aliquots of the lipid dispersion were pipetted into 3-ml (measured volume) serum glass vials (item \#223683, Wheaton, Millville, NJ, USA) and sealed using 13-mm butyl rubber stoppers (part \#W224100-093, Wheaton) and 13-mm aluminum crimps (part \#224182-01, Wheaton). Similarly, aliquots of the buffer solution (PBS:propylene glycol:glycerol = 16:3:1 v/v) were stored in 3-ml serum vials, sealed using butyl rubber stoppers, and crimped. The air in the headspace of each vial (containing either the lipid dispersion or buffer solution only) was evacuated using a vacuum pump (model number: 8803, Welch Vacuum Technologies Inc., Mt. Prospect, IL, USA) for $30 \mathrm{~s}$ to a pressure of $40 \mathrm{mmHg}$. The vials were stored at $4{ }^{\circ} \mathrm{C}$ and used for experiments within 2 weeks of preparation.

Before use, each vial was taken from the refrigerator and allowed to reach room temperature for $1 \mathrm{~h}$. Xenon and OFP gas were collected in gas-impermeable 0.5-1 Tedlar® bags (Zefon International, Ocala, FL, USA). The headspace of each vial $(1.6 \mathrm{ml})$ was filled with either Xe or a mixture of Xe and OFP $(9: 1 v / v)$ using a 3-ml syringe equipped with a $30-\mathrm{G}$ needle. Subsequently, Xe-MB and Xe-OFP-MB were prepared by activating the vial by high-shear mixing for $45 \mathrm{~s}$ with a VIALMIX ${ }^{\mathrm{TM}}$ device (Lantheus Medical Imaging, North Billerica, MA, USA). The microbubble vials were allowed to cool for $15 \mathrm{~min}$ to room temperature before use.

\section{Size Distribution Assessment}

The size distributions of Xe-MB and Xe-OFP-MB were measured at $25^{\circ} \mathrm{C}$ using a Coulter counter (Multisizer 4, Beckman Coulter, Brea, CA, USA) equipped with a $30-\mu \mathrm{m}$ aperture [34]. Xe-MB and Xe-OFP-MB were diluted in PBS 830fold and 2300-fold, respectively, prior to measuring the size distributions. The PBS was either a) Xe-saturated, b) air-saturated, or c) undersaturated (dissolved oxygen $=87.5 \pm 1.8 \%$ ). Xenon-saturated solution mimicked "in vial" conditions. Undersaturated solutions mimicked total venous gas saturation levels [35]. A dissolved oxygen (DO) meter (FirestingO2Pyroscience, PyroScience GmbH, Aachen, Germany) was 
used to measure the DO as a surrogate metric for total dissolved gas saturation [36].

For each measurement, $100 \mu \mathrm{l}$ of the diluted sample was analyzed using a $30-\mu \mathrm{m}$ aperture. Three replicate measurements were performed for each vial within 2 min of dilution, and 3 independent vials were assessed. A volume-weighed density histogram with 200 linearly spaced bins spaced between 0.6 and $18 \mu \mathrm{m}$ was obtained and adjusted for the dilution in PBS. The total volume was computed by summing the volume measured in all bins between 0.6 and $18 \mu \mathrm{m}$.

\section{Broadband Acoustic Attenuation Measurements}

The frequency-dependent acoustic attenuation coefficients of Xe-MB, Xe-OFP-MB, and Definity ${ }^{\circledR}$ were measured using through-transmission attenuation spectroscopy as described previously [34, 37]. Xe-MB, Xe-OFP-MB, or Definity ${ }^{\circledR}$ was diluted in a $37 \pm 0.5{ }^{\circ} \mathrm{C}$ solution of $0.5 \%$ BSA in airsaturated PBS (DO $=99.6 \pm 0.5 \%)$. The lipid concentration of Definity ${ }^{\circledR}$ is $0.75 \mathrm{mg} / \mathrm{ml}$, whereas the lipid concentration of Xe-MB and Xe-OFP-MB is $1 \mathrm{mg} / \mathrm{ml}$. To attain equivalent lipid concentration, the attenuation coefficient of Definity ${ }^{\circledR}$ was measured at a dilution of 1:1500, and Xe-MB and XeOFP-MB were diluted 1:2000 (v/v). The attenuation spectroscopy system (Fig. 1) consisted of a reservoir, a rectangular sample chamber that had acoustically transparent polycarbonate film windows (CLINIcell ${ }^{\circ}$ 25, Mabio, Tourcoing, France), and 2 transducers mounted in an acrylic tank filled with deionized water maintained at $37.0 \pm 0.5^{\circ} \mathrm{C}$ using a circulating water bath (Neslab EX, Newington, NH, USA). A diluted dispersion of Xe-MB, Xe-OFP-MB, or Definity® was gravity-fed from the reservoir into the rectangular sample chamber. Two broadband transducers (PI-20, Olympus NDT, Waltham, MA, USA) were used to measure the attenuation spectrum over the frequency range of 2 to $25 \mathrm{MHz}$, immediately after the dilution of the agent. Three independent vials were used ( 1 measurement per vial) for all attenuation measurements. The same system was used along with 6-MHz/ 220-kHz ultrasound insonation to assess ultrasound-triggered release of gas payload from Xe-MB and Xe-OFP-MB $(n=3$ vials, 1 measurement per vial), as described in the "Quantification of Xe dose" section.

\section{Effect of Dissolved Gas Content on Microbubble Stability}

The effect of the dissolved gas content on the stability of Xe$\mathrm{MB}$ and Xe-OFP-MB was assessed using attenuation spectroscopy at $37.0 \pm 0.5^{\circ} \mathrm{C}$. Xe-MB and Xe-OFP-MB were diluted $1: 2000(v / v)$ in a $0.5 \%$ BSA solution with a DO maintained at $87.5 \pm 1.8 \%$, to mimic total gas saturation of venous blood [35]. The frequency-dependent acoustic attenuation coefficient was measured for each sample as described in the previous section.

\section{Ultrasound Imaging}

In vitro ultrasound imaging of a flow phantom comprised of a latex tube (6-mm inner diameter) was performed using a Verasonics scanner (Verasonics, Inc., Kirkland, WA, USA) and an L7-4 linear array transducer (5.2-MHz center frequency, Philips, Bothell, WA, USA). Baseline images of the tube filled with only PBS were acquired. Subsequently, Definity ${ }^{\circledR}$ was diluted 3000-fold and Xe-MB and Xe-OFPMB were diluted 4000-fold (to maintain equivalent lipid concentrations) and infused into the flow phantom at a flow rate of $5 \mathrm{ml} / \mathrm{min}$. B-mode cine loops comprising 20 frames were recorded. The echogenicity of the perfused regions was quantified using a custom script in MATLAB® (The Mathworks, Natick, MA, USA).
Fig. 1 Schematic of the attenuation spectroscopy system and the experimental setup used for evaluating the ultrasoundtriggered release of $\mathrm{Xe}$ from $\mathrm{Xe}$ OFP-MB. Tx $=$ transmitting transducer; $\mathrm{Rx}=$ receiving transducer

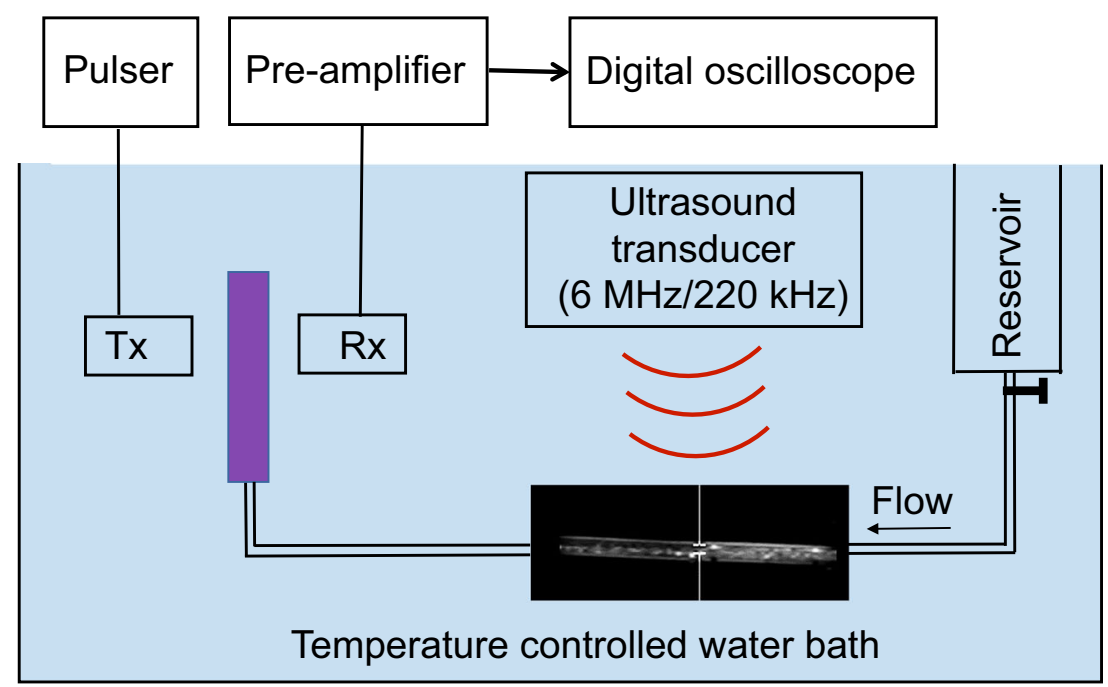


In vivo imaging was performed in an inbred strain of male C57 mouse (28 g weight). Xenon-loaded microbubbles were injected retrograde in the internal jugular vein, and highfrequency B-mode imaging was performed. The mouse was given buprenorphine $\mathrm{HCl}$ subcutaneously as a pre-emptive analgesic $(0.1 \mathrm{mg} / \mathrm{kg})$, anesthetized by inhalation of $2.5 \%$ isoflurane, and surgically prepared. An incision was made on the ventral part of the neck and on the dorsal side between the scapulae. A pulled polyurethane catheter (MRE 040, Braintree Scientific, Braintree, MA, USA) with an outer diameter of $0.4 \mathrm{~mm}$ was placed in the right jugular vein and anchored with ligatures. The catheter was tunneled out of the back, flushed with $100 \mathrm{U}$ heparin, and capped with a 21$\mathrm{G}$ stainless steel plug. The mouse was prepared for contrastenhanced echocardiographic imaging of Definity ${ }^{\circledR}$, Xe-OFP$\mathrm{MB}$, and Xe-MB. The chest hair of the mouse was removed using a depilatory cream (Nair, Church and Dwight, Ewing, NJ, USA) and placed on an imaging platform. The mouse was maintained at 36 to $37{ }^{\circ} \mathrm{C}$ with the use of a heated platform and lamp. B-mode imaging was performed using a Vevo 2100 imaging system (VisualSonics, Toronto, ON, Canada) equipped with a MS250 probe (13-24 MHz bandwidth) operating at an 18-MHz center frequency. Acoustic parameters were set to $10 \%$ output power and $25 \mathrm{~dB}$ gain. Definity ${ }^{\circledR}$, $\mathrm{Xe}-\mathrm{OFP}-\mathrm{MB}$, and Xe-MB were infused into the mouse 1 by 1 , and cine loops were recorded. For all agents, a $10-\mu$ l bolus of the diluted agent was injected into the mouse using a blunt 22-gauge needle coupled to the indwelling catheter. Definity® was diluted 1:10-fold in sterile, air-saturated saline $(0.9 \%)$, and Xe-OFP-MB and Xe-MB were diluted 1:13.33-fold in xenon-saturated saline to obtain equivalent lipid concentration. The catheter was flushed between doses with approximately $15-\mu l$ saline. The pretrigger mode was used to destroy residual microbubbles with high-amplitude ultrasound, and atleast $10 \mathrm{~min}$ was allowed between infusion of different agents to allow the circulating microbubbles to be cleared by the reticuloendothelial system and lungs. The perfused region was segmented, and the echogenicity was quantified using the VevoCQ software (VisualSonics, Toronto, ON, Canada).

\section{Quantification of Xe Dose}

The quantification of Xe and OFP dose in the microbubbles was performed using GC/MS [38]. The Xe-MB and Xe-OFP-MB vials were shipped on ice overnight to the University of Texas Health Science Center at Houston, and GC/MS was performed on the second day after adding xenon or xenon and OFP gas to the vials through the septum. Additionally, the shelf life of Xe$\mathrm{MB}$ and Xe-OFP-MB was assessed using GC/MS at 2 days, 7 days, 14 days, and 28 days after filling the vial headspace with Xe $(100 \%)$ or Xe and OFP $(90 \% / 10 \% v / v)$, respectively. At least 3 vials were used for the measurement on each day (1 measurement per vial). The GC/MS system consists of a mass spectrometer (HP 5973A, Agilent Technologies, Santa Clara, CA, USA) interfaced with a gas chromatograph (HP 6890, Agilent Technologies), a G1512 controller, GC injector (Tower and Tray, Agilent Technologies), and a rough pump (E2M2, Edwards, Burgess Hill, West Sussex, UK). This instrument was controlled using software (G1701 DA, Agilent Technologies) installed on a dedicated computer. Gases were separated using an Agilent CP-Molsieve 5A column (25-m length, $0.32-\mathrm{mm}$ inner diameter, $30-\mu \mathrm{m}$ film thickness) that was interfaced with the mass spectrometer through identical particle traps (CP7536PT, Agilent J\&W, Wilmington, DE, USA).

Xenon and OFP gases were removed from compressed gas cylinders with a syringe and transferred to 2-ml glass sampling vials (filled with air), sealed with Teflon-lined screw-top caps. Percent standards $(v / v)$ were produced by mixing either Xe or $\mathrm{Xe}(90 \%)$ and OFP (10\%) mixture with air to attain concentrations of 1.25 to $10 \%$. Aliquots of $5 \mu$ l were injected manually into the GC system from the 2-ml sampling vials using an airtight syringe (SGE Syringe, 10R-GT-LC, RESTEK, Australia). Three independent vials were used for performing the standard measurements ( 1 measurement per vial). The molecular sieve column eluted Xe between 7.5 and 9 min and OFP between 1.1 and $1.6 \mathrm{~min}$ (Fig. 2). The peak for Xe and OFP occurred at 7.64 and $1.19 \mathrm{~min}$, respectively. The area under the peak corresponding to either Xe or OFP was determined in auto-integration mode. Linear regression was performed to obtain a calibration curve relating the volume of Xe in the sample headspace with measured peak areas (Fig. 3). A calibration curve for OFP measurements (not shown) was also created using the same procedure. The calibration curves of Xe diluted in air and xenon and OFP diluted in air were represented by the equations: $y=1.5 \times 10^{9} x+7.1 \times 10^{6}$ and $y=1.3 \times 10^{9} x-2.1 \times$ $10^{6}$, respectively, where $\mathrm{x}$ represents the Xe dose in microliters and $y$ represents the area under the curve. Similarly, the calibration curve for OFP diluted in air was represented by the equation: $y=6.0 \times 10^{8} x-4.3 \times 10^{5}$.

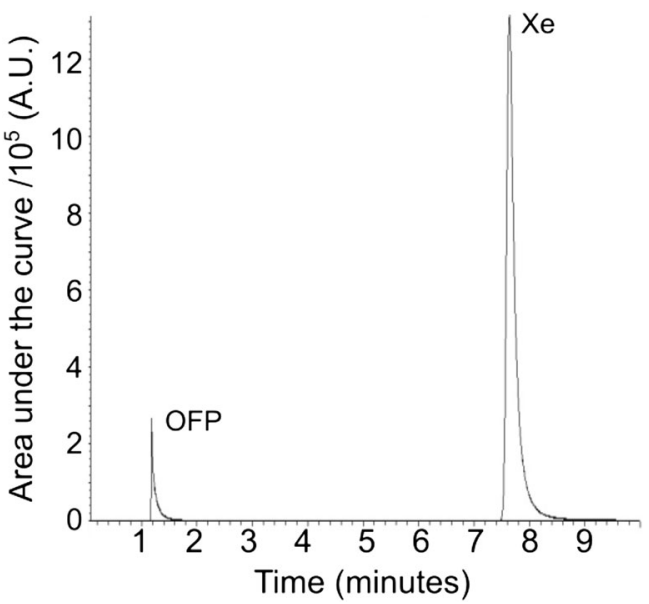

Fig. 2 A representative GC/MS chromatogram showing separation of Xe and OFP by the molecular sieve 


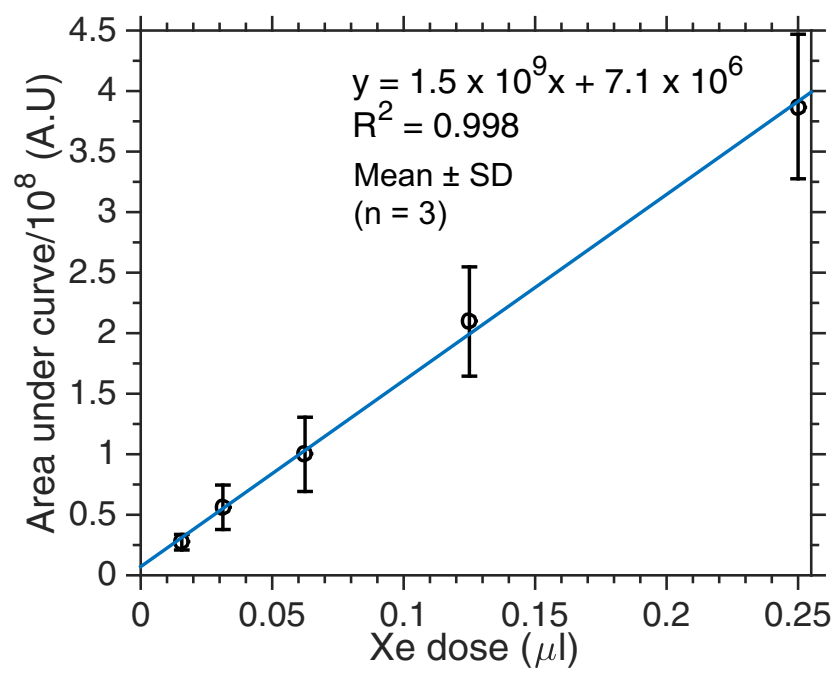

Fig. 3 Calibration curve for Xe gas obtained from gas chromatography/ mass spectrometry. The area under the curve is plotted against the $\mathrm{Xe}$ dose in a xenon-air mixture of a fixed volume $(5 \mu \mathrm{l})$

To determine the Xe dose in Xe-MB and Xe-OFP-MB, the microbubbles were redispersed by inverting the vial gently. Thereafter, $500 \mu \mathrm{l}$ of the dispersion was aliquoted to a fresh 2-ml glass vial (66020-950, VWR, Radnor, PA, USA). The vial was sonicated for $5 \mathrm{~min}$ to release Xe from microbubbles and to accelerate the equilibration of Xe into the headspace by convectivefluid mixing. Using a $10-\mu l$ gastight syringe (SGE Syringe, 10R-GT-LC, RESTEK, Australia), 5- $\mu$ l gas was drawn from the headspace and injected manually into the GC/MS system. Four vials were used for measurements with both Xe-MB and Xe-OFP-MB. The volume of Xe dissolved in Xe-saturated and Xe-OFP-saturated solution (16:3:1 v/v PBS:propylene glycol:glycerol) was measured using the same protocol. Three vials of Xe-saturated and Xe-OFP-saturated solution were tested.

The volume of $\mathrm{Xe}$ in the headspace was calculated using the calibration curve and reported in terms of microliters of Xe per milligram of lipid. Note that the concentration of lipid in Xe-MB and Xe-OFP-MB was $1 \mathrm{mg} / \mathrm{ml}$. The volume of Xe was also determined experimentally by sampling the vial headspace sequentially between 0 and $18 \mathrm{~h}$ (without sonication) to assess the time taken for xenon gas to diffuse through the solution and equilibrate with the headspace. The total $\mathrm{Xe}$ dose in $\mathrm{Xe}-\mathrm{MB}$ and Xe-OFP-MB was also computed by summing the expected volume of $\mathrm{Xe}$ dissolved in solution (16:3:1 v/v PBS:propylene glycol:glycerol) and lipid [39] (based on published solubility coefficients), with the volumes measured using the Coulter counter. The presence of dissolved salts and glycerol is expected to reduce the solubility of Xe in solution by $9.3 \%$ [40] and 5.7\% [41] relative to water, respectively. At the concentrations used in this study, propylene glycol is not expected to affect the solubility of Xe relative to the aqueous solution [42]. Based on the partition coefficient of $\mathrm{Xe}$ in aqueous solutions 0.089 [43], headspace analysis is expected to underestimate the measured dose of Xe by $8.2 \%$.

\section{Ultrasound-Triggered Gas Payload Release}

As a step toward in vivo experiments, the feasibility of ultrasound-triggered payload release was assessed using ultrasound parameters relevant for vascular and transcranial applications (6-MHz and $220-\mathrm{kHz}$ frequencies, respectively). The $\mathrm{Xe}-\mathrm{MB}$ or Xe-OFP-MB were diluted $(1: 2000 v / v)$ in $0.5 \%$ BSA solution in a flow system maintained at $37.0 \pm 0.5{ }^{\circ} \mathrm{C}$ (Fig. 1). A decrease in the attenuation coefficient upon ultrasound exposure served as an indicator of gas release from Xe$\mathrm{MB}$ and Xe-OFP-MB. Three vials of either Xe-MB and XeOFP-MB were used for each group (no ultrasound, ultrasound at mechanical index $(\mathrm{MI})=0.03$, and ultrasound at $\mathrm{MI}=0.8$ ).

To evaluate microbubble destruction using ultrasound, a clinical scanner (HDI 5000, Philips, Bothell, WA, USA) equipped with an L12-5 linear array probe was used. The diluted Xe-MB and Xe-OFP-MB were perfused into a 1$\mathrm{mm}$ inner diameter ethyl vinyl acetate tube (McMasterCarr, Aurora, OH, USA) using a syringe pump (Pump 11 Elite Infusion/Withdrawal Programmable Single Syringe, Harvard Apparatus, Holliston, MA, USA) in withdrawal mode. The tube diameter and flow rate $(5 \mathrm{ml} / \mathrm{min})$ mimicked the time-averaged volumetric flow in a rat carotid artery [ 44 , 45]. The transducer was placed at a distance of $1 \mathrm{~cm}$ from the tube. The tube was insonified using either duplex Doppler ultrasound at $6-\mathrm{MHz}$ frequency and $\mathrm{MI}$ of 0.8 or B-mode $(\mathrm{MI}=0.03)$. These experiments were performed within $20 \mathrm{~s}$ of agent dilution. Note that $6-\mathrm{MHz}$ frequency is used typically for carotid imaging applications.

Ultrasound-triggered gas release was also evaluated at $220 \mathrm{kHz}$ using a single-element piezocomposite transducer (38-mm aperture), as a step toward Xe delivery for treating intracerebral hemorrhage. Of note, using $220-\mathrm{kHz}$ frequency allows adequate penetration of acoustic energy through the skull [46]. Xe-MB or Xe-OFP-MB were diluted in 2-ml PBS and transferred to the CLINIcell ${ }^{\circledR}$, forming a fluid column $(3.8 \mathrm{~cm}$ width $\times 0.75 \mathrm{~cm}$ height $\times 0.5 \mathrm{~cm}$ depth $)$ which only partially filled the CLINIcell@. The CLINIcell ${ }^{\circledR}$ was placed $5 \mathrm{~cm}$ from the transducer and exposed to either ultrasound (15-cycle pulses at $0.44 \mathrm{MPa}$ peak-to-peak pressure, $5-\mathrm{kHz}$ pulse repetition frequency, $\mathrm{MI}=0.47$ ) or sham (no ultrasound) for $10 \mathrm{~s}$, following which the attenuation was measured. This static condition was chosen to model an occluded middle cerebral artery $[47,48]$. These measurements were performed within $20 \mathrm{~s}$ of agent dilution in PBS, which is comparable to the murine blood recirculation time [49]. 


\section{Statistical Analysis}

The data are reported as the mean \pm 1 standard deviation (S.D.). The Mann-Whitney nonparametric test was used to determine whether the differences in particle volume and payload of Xe in Xe-MB or Xe-OFP-MB were statistically significant. Statistical analysis was performed using GraphPad Prism 7 (GraphPad Software Inc., La Jolla, CA, USA). A $p$ value of less than 0.05 was considered statistically significant.

\section{Results}

\section{Size Analysis of Xe-MB and Xe-OFP-MB}

The number- and volume-weighted size distributions of XeMB and Xe-OFP-MB are shown in Fig. 4a, b. The "in vial"

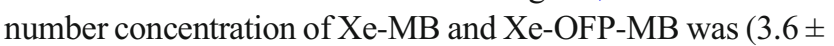
$1.3) \times 10^{9}$ and $(5.9 \pm 0.3) \times 10^{9}$ microbubbles $/ \mathrm{ml}$, respectively. Also, over $94.2 \%$ of Xe-MB and over $99.7 \%$ of Xe-OFPMB were smaller than $10 \mu \mathrm{m}$. For comparison, $99.9 \%$ of Definity® particles were smaller than $10 \mu \mathrm{m}$ ([37]). The total volume of Xe-MB was the highest when measured in Xesaturated PBS $(34.5 \pm 4.4 \mu \mathrm{l} / \mathrm{ml})$ and decreased for airsaturated $(19.9 \pm 12.2 \mu \mathrm{l} / \mathrm{ml})$ and undersaturated PBS $(1.3 \pm$ $0.2 \mu \mathrm{l} / \mathrm{ml})(p<0.01)$. The total volume of Xe-OFP-MB was not different when measured in Xe-saturated, air-saturated, and undersaturated PBS $(p>0.05)$. Measurements in Xesaturated water revealed that the total volume of gas payload encapsulated in Xe-OFP-MB $(83.7 \pm 10.1 \mu \mathrm{l} / \mathrm{ml})$ was 2.4 -fold higher than that in Xe-MB $(p<0.01)$. Table 1 lists the total dose of xenon in Xe-MB and Xe-OFP-MB solutions obtained by summing the gas payload measured with the Coulter counter and adding the amount calculated in the lipid shells and in the Xe-saturated or Xe-OFP-saturated solutions [39-41] using Raoult's law [50]. For this total Xe dose calculation, the Xe$\mathrm{MB}$ or Xe-OFB-MB volume measured in the presence of Xesaturated or Xe-OFP-saturated solution was considered instead of air-saturated or undersaturated solution to mimic the conditions during GC/MS measurements.

\section{Attenuation Coefficient of Xe-MB, Xe-OFP-MB, and Definity ${ }^{\circledR}$ (2-25 MHz)}

The attenuation coefficient of Xe-MB, Xe-OFP-MB, and Definity ${ }^{\circledR}$ measured in $0.5 \%$ BSA solution $(100 \pm 1 \%$ DO) immediately after dilution is shown in Fig. 5. The dashed and dotted vertical lines denote 5.2 and $18 \mathrm{MHz}$, the frequencies used for subsequent B-mode imaging experiments. The attenuation coefficient for Xe-OFP-MB was up to $50 \mathrm{~dB}$ higher than Xe-MB. Xe-MB showed an attenuation coefficient $9.2 \mathrm{~dB} / \mathrm{cm}$ at $5.2 \mathrm{MHz}$, but almost no attenuation at $18 \mathrm{MHz}$. Xe-OFP-MB demonstrated similar attenuation to Definity ${ }^{\circledR}(29 \mathrm{~dB})$ at $5.2 \mathrm{MHz}$, whereas its attenuation was $8 \mathrm{~dB}$ lower than Definity ${ }^{\circledR}$ at $18 \mathrm{MHz}$.

The attenuation coefficient of Xe-MB and Xe-OFP-MB in undersaturated $\mathrm{BSA}$ solution ( $\mathrm{DO}=87.5 \pm 1.8 \%$ ) is illustrated in Fig. 6. The attenuation coefficient of Xe-MB approached 0, indicating a complete release of the gas payload in undersaturated BSA solution. However, the Xe-OFP-MB demonstrated an attenuation coefficient of up to $75 \mathrm{~dB} / \mathrm{cm}$ in undersaturated BSA solution.

\section{Ultrasound Imaging}

Figure $7 \mathrm{a}-\mathrm{d}$ shows B-mode images of a latex tube $(6-\mathrm{mm}$ diameter) (a) before administration of contrast agent, after infusion of (b) Xe-MB, (c) Xe-OFP-MB, and (d) Definity®. Figure $7 \mathrm{e}-\mathrm{h}$ shows high-frequency B-mode images of the mouse left ventricle (e) before administration of contrast agent (baseline) and after administration of (f) Xe-MB, (g) Xe-OFP$\mathrm{MB}$, and (h) Definity®. At $5.2 \mathrm{MHz}$ (in vitro imaging), Xe$\mathrm{MB}, \mathrm{Xe}-\mathrm{OFP}-\mathrm{MB}$, and Definity® produced a 24-, 29-, and 33-dB enhancement relative to baseline, respectively. At $18 \mathrm{MHz}$ (in vivo imaging) Xe-OFP-MB and Definity ${ }^{\circledR}$ produced a 13- and 27-dB enhancement in echogenicity relative

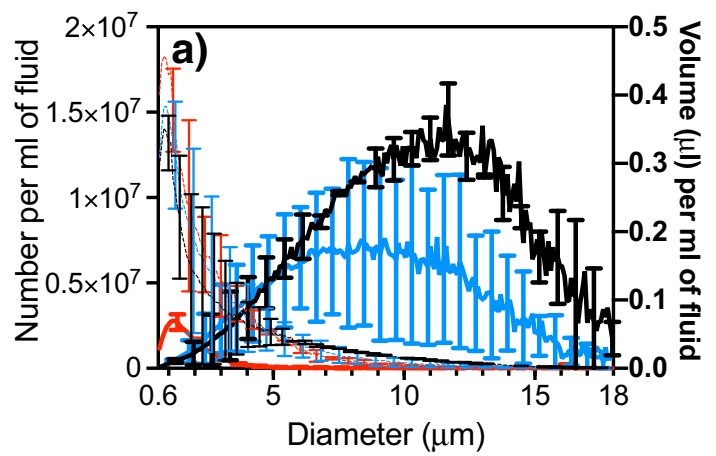

Fig. 4 The number-weighted (left ordinate, denoted by thin dotted lines) and volume-weighted (right ordinate, denoted by thick solid lines) size distributions of (a) Xe-MB and (b) Xe-OFP-MB, measured in Xe-

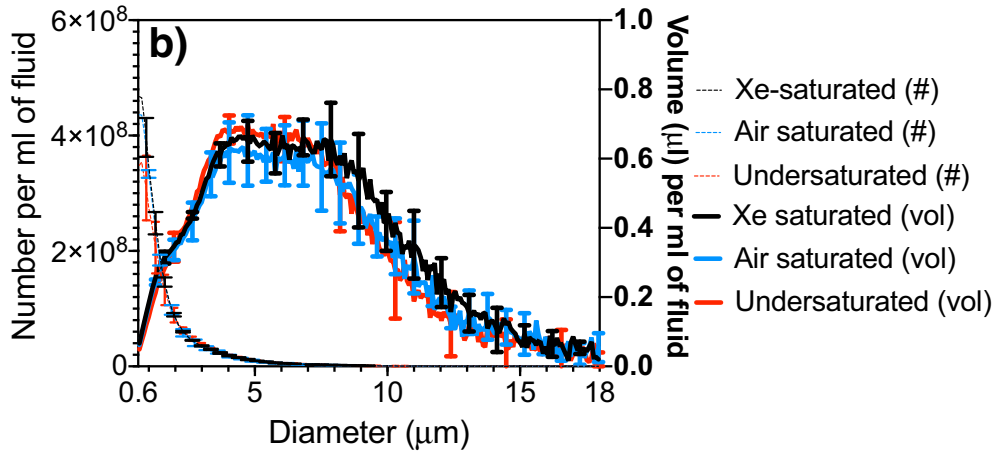

saturated, air-saturated, and undersaturated $(\mathrm{DO}=87.5 \pm 1.8 \%)$ PBS. Mean $\pm \operatorname{SD}(\mathrm{n}=3)$ 
Table 1 Xenon concentration estimated using Coulter counter measurements and solubility coefficients of Xe in lipid [39] and aqueous solution [40]

\begin{tabular}{lllll}
\hline Agent & {$[\mathrm{Xe}](\mu \mathrm{l} / \mathrm{ml}$ of solution, mean \pm S.D.)* } & & \\
\cline { 2 - 5 } & $\begin{array}{l}\text { Coulter counter } \\
\text { measurement }\end{array}$ & $\begin{array}{l}\text { Calculated } \\
\text { aqueous }\end{array}$ & $\begin{array}{l}\text { Calculated } \\
\text { lipid }\end{array}$ & Total \\
\hline Xe-MB & $34.5 \pm 4.4$ & 75.5 & 1.9 & $110.4 \pm 4.4(n=3)$ \\
Xe-OFP-MB & $83.7 \pm 10.1$ & 66.7 & 1.7 & $151.4 \pm 10.1(n=3)$ \\
\hline
\end{tabular}

*The Xe dose per milliliter of solution is equivalent to the Xe dose per milligram of lipid to the baseline, respectively. However, no enhancement ( $0 \mathrm{~dB})$ was observed for Xe-MB. The duration of contrast enhancement was nearly $90 \mathrm{~s}$ for Definity and $30 \mathrm{~s}$ for Xe-OFP-MB. No noticeable change was observed in breathing and heart rate after injecting the agents in the mouse, suggesting that the dose was well tolerated.

\section{Xenon Quantification Using GC/MS}

Table 2 shows the Xe dose per milliliter of solution in Xe-MB, $\mathrm{Xe}-\mathrm{OFP}-\mathrm{MB}$ and Xe-saturated solution, and Xe-OFPsaturated solution. For comparison, the $\mathrm{Xe}$ dose in $\mathrm{Xe}$ saturated and Xe-OFP-saturated solutions (without microbubbles) is also listed. Xe-MB and Xe-OFP-MB carried a higher dose than Xe-saturated or Xe-OFP-saturated solutions $(p<0.01)$. Note that $1 \mathrm{ml}$ of Xe-MB or Xe-OFP-MB solution contains $1 \mathrm{mg}$ of lipid. Therefore, the Xe dose per milliliter of solution is equivalent to the Xe dose per milligram of lipid. The Xe dose in Xe-OFP-MB was significantly ( $p=$ 0.014 ) higher by $28.7 \pm 28.8 \%$ than in Xe-MB. The measured $\mathrm{Xe}$ dose in Xe-saturated and Xe-OFP-saturated solution were not different $(p>0.05)$. It took over $4 \mathrm{~h}$ for Xe in the solution to equilibrate with the headspace (data not shown). The

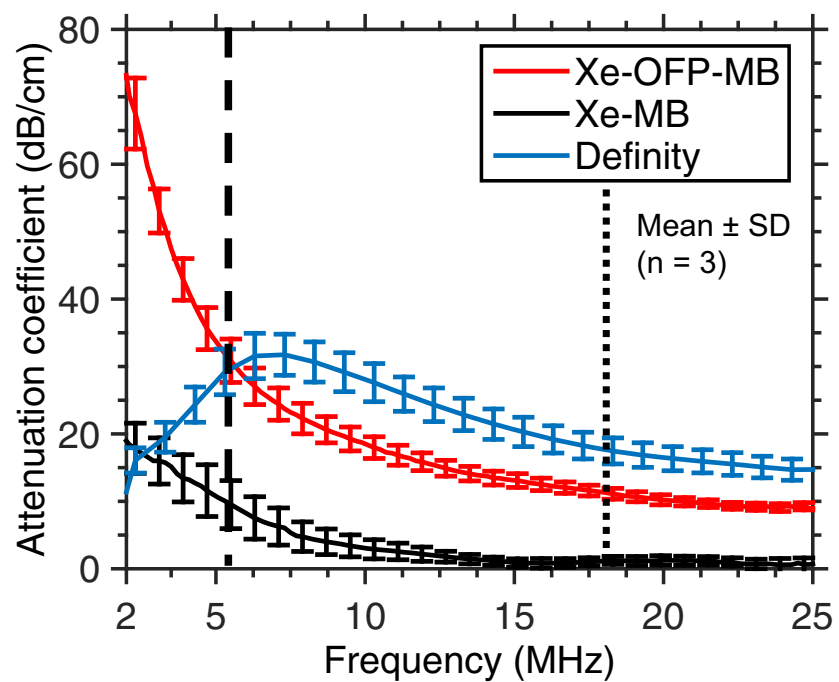

Fig. 5 The frequency-dependent acoustic attenuation coefficient of Xe$\mathrm{MB}, \mathrm{Xe}-\mathrm{OFP}-\mathrm{MB}$, and Definity ${ }^{\circledR}$ measured in BSA solution (dissolved gas $=100 \pm 1 \%$ ) at $37^{\circ} \mathrm{C}$. The dashed and dotted vertical lines denote the frequency of subsequent in vitro and in vivo imaging experiments measured percentage of Xe and OFP in Xe-OFP-MB corresponded to $89.1 \pm 28.1 \%$ and $15.5 \pm 7.5 \%$ of the total gas volume, which is consistent with the percentage of Xe and OFP loaded in the headspace before high-shear mixing ( $90 \%$ and $10 \%$, respectively).

The results of shelf-life assessment up to 28 days are shown in Fig. 8. These results show nearly a $30 \%$ decrease in the measured Xe gas payload over 1 week for Xe-MB and no change for Xe-OFP-MB. Beyond 7 days, the Xe dose in Xe$\mathrm{MB}$ and Xe-OFP-MB continued to decrease progressively until 28 days. Specifically, at days 7, 14, and 28, Xe-MB lost $29.1 \%, 51.8 \%$, and $68.1 \%$ of the Xe dose relative to measurements on day 2. However, the corresponding decrease in Xe dose of Xe-OFP-MB was only $0.6 \%, 18.2 \%$, and $47.8 \%$ at the same time points.

\section{Ultrasound-Triggered Gas Payload Release}

The attenuation coefficients of Xe-MB and Xe-OFP-MB measured with and without exposure to 6-MHz duplex Doppler or B-mode ultrasound are illustrated in panels $a$ and $b$ in Fig. 9, respectively. The attenuation coefficients measured without ultrasound exposure and with B-mode ultrasound $(\mathrm{MI}=0.03)$ exposure were similar. Both Xe-MB and Xe-OFP-MB

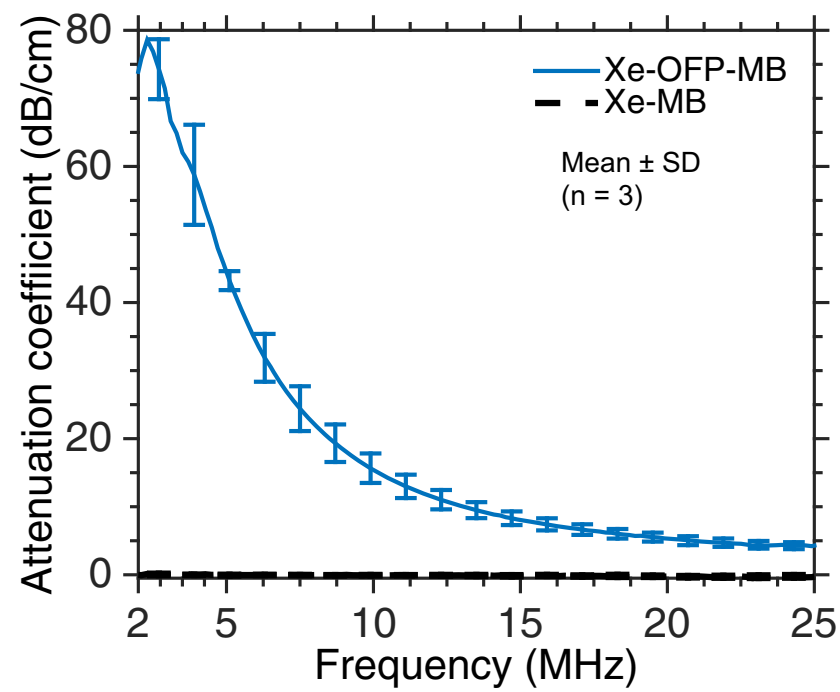

Fig. 6 The frequency-dependent attenuation coefficient of Xe-MB and $\mathrm{Xe}-\mathrm{OFP}-\mathrm{MB}$ in undersaturated $\mathrm{BSA}$ solution $(\mathrm{DO}=87.5 \pm 1.8 \%)$ 

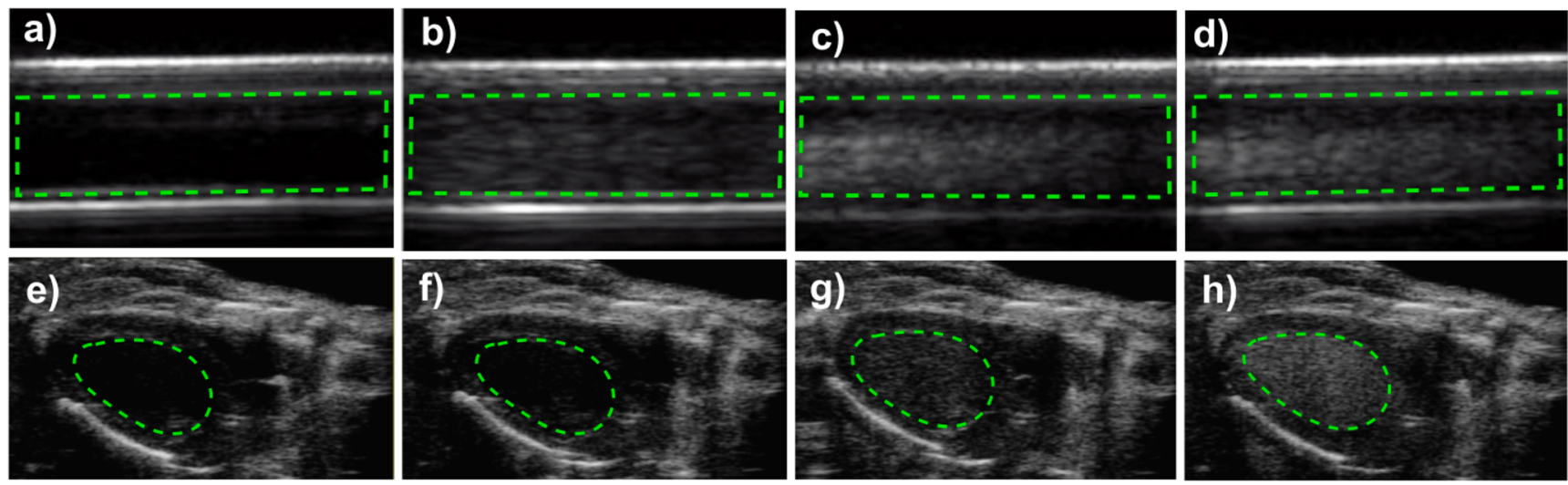

Fig. 7 (a-d) B-mode images of a latex tube (6-mm diameter) (a) before administration of contrast agent, after infusion of (b) Xe-MB, (c) XeOFP-MB, and (d) Definity®. (e-h) Parasternal long axis view of the left ventricle of a mouse: (e) baseline image without contrast agent, (f)

with Xe-MB, (g) with Xe-OFP-MB, and (h) with Definity®. The regions of interest for determining echogenicity are indicated by dotted green lines

remained attenuative when insonified with B-mode pulses. However, a marked decrease in the attenuation coefficient was observed after insonification with spectral duplex Doppler $(\mathrm{MI}=0.8)$. The attenuation coefficients of Xe-MB and Xe-OFP-MB with and without exposure to 220-kHz pulsed ultrasound are shown in panels a and b in Fig. 10, respectively. Almost complete loss of attenuation was observed after exposure to $220-\mathrm{kHz}$ pulsed ultrasound $(\mathrm{MI}=0.47)$.

\section{Discussion}

The findings of this study demonstrate that a) Xe can be loaded in lipid-shelled microbubbles that are stabilized against dissolution by co-encapsulation of OFP, b) co-encapsulation of OFP enhanced the Xe payload and contrast produced by lipid-shelled microbubbles, and c) ultrasound can be used to release the Xe payload from microbubbles, using parameters suitable for carotid and transcranial insonation. The microbubbles used in the present study were prepared using DSPC and 18:0 PEG2000 PE. These lipids have long acyl

Table 2 Xenon concentration measured using gas chromatography/ mass spectrometry. Seven vials were tested for Xe-MB, 8 vials for XeOFP-MB, and 3 vials each for Xe-saturated and Xe-OFP-saturated solution, respectively

\begin{tabular}{ll}
\hline Agent & {$[\mathrm{Xe}](\mu \mathrm{l} / \mathrm{ml} \text { of solution, mean } \pm \text { S.D. })^{*}$} \\
\hline Xe-MB & $113.1 \pm 13.5$ \\
Xe-OFP-MB & $145.6 \pm 25.5$ \\
Xe-saturated solution & $34.1 \pm 2.7$ (with sonication) \\
& $57.6 \pm 4.2(8 \mathrm{~h}$ equilibrium) \\
Xe-OFP-saturated solution & $39.1 \pm 2.1$ (with sonication) \\
& $51.0 \pm 3.3(8 \mathrm{~h}$ equilibrium)
\end{tabular}

*The Xe dose per milliliter of solution is equivalent to the Xe dose per milligram of lipid

chains that improve the stability of microbubbles [51]. Specifically, DSPC lowers the surface tension [52] and resists surface shear [53] and gas diffusion [54]. PEG2000 PE serves as an emulsifier and forms hydrated PEG brushes that prevent microbubble coalescence [55].

The measured volume of Xe-MB in Xe-saturated PBS was higher than air-saturated and undersaturated PBS because of a reduction in the diffusion gradient, which could enhance the propensity of Xe to remain encapsulated in the microbubbles. The higher stability of Xe-MB in an air-saturated solution relative to the undersaturated solution is consistent with a previously reported study with SonoVue ${ }^{\circledR}$, a lipid-shelled ultrasound contrast agent filled with sulfur hexafluoride. Specifically, SonoVue ${ }^{\circledR}$ microbubbles dispersed in $100 \%$ air-saturated water persisted 6-fold longer than in $80 \%$ saturated water [36]. The size distribution studies suggest that diluting the agent in Xe-saturated PBS stabilizes the Xe payload. Undiluted microbubbles are dispersed in Xe-saturated solution, which will keep the payload stable. Thus, the agent

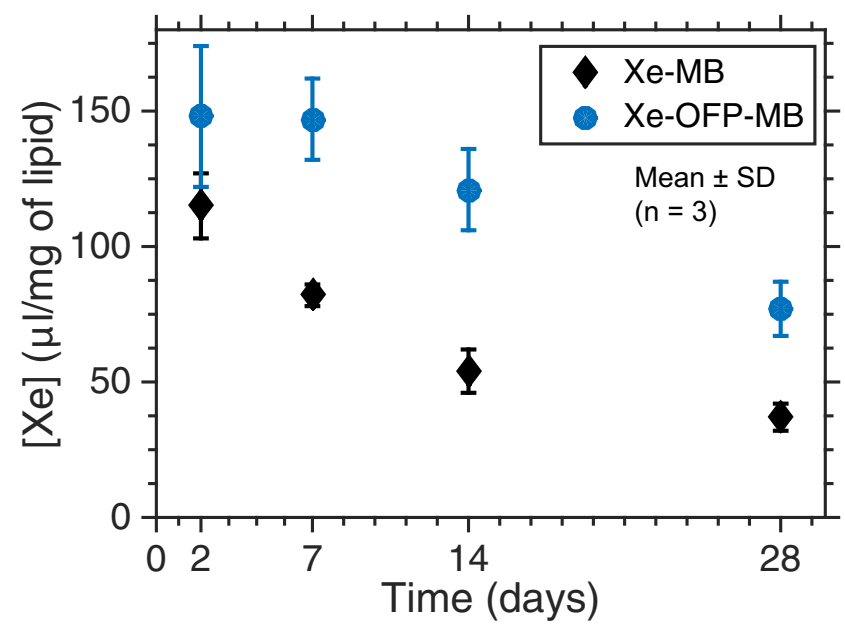

Fig. 8 The dose of $\mathrm{Xe}$ in Xe-MB and Xe-OFP-MB measured up to 28 days 


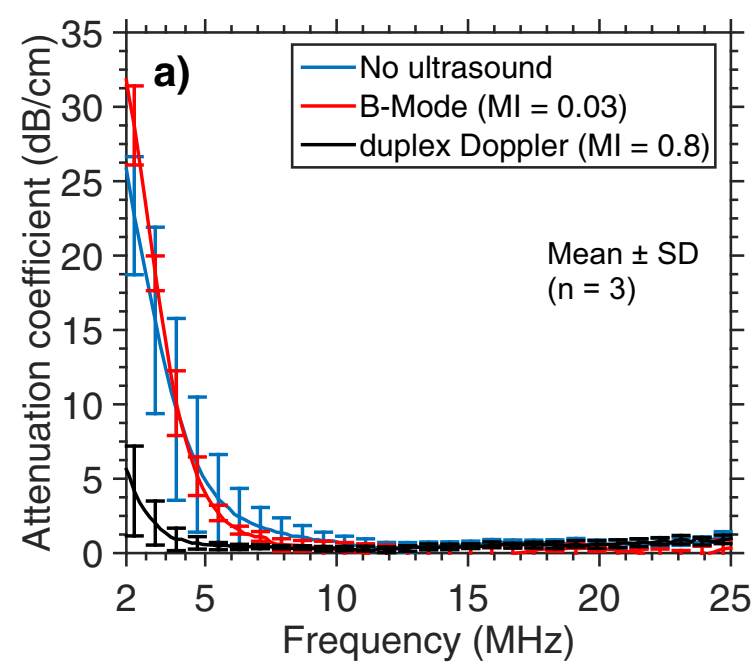

Fig. 9 Ultrasound-triggered attenuation loss using duplex Doppler ultrasound (6-MHz) for (a) Xe-MB and (b) Xe-OFP-MB. Attenuation coefficients as a function of frequency for (a) Xe-MB and (b) Xe-OFP-

could be administered in vivo as an undiluted bolus, or after dilution in Xe-saturated saline. Additionally, the volumeweighted size distributions demonstrated an increase in the volume of microbubbles when OFP was combined with Xe. Xenon has a higher aqueous solubility than OFP (Ostwald solubility constants at $37^{\circ} \mathrm{C}$ are $8.9 \times 10^{-2}$ and $5.2 \times 10^{-4}$, respectively $[43,56]$. The osmotic action of OFP due to its high vapor pressure and low aqueous solubility [32] could have contributed to the stability of Xe-OFP-MB. In addition, perfluorocarbon gases may reduce the surface tension by intercalating in the lipid monolayer [31, 57]. The presence of OFP during high-shear mixing can promote the formation of stable microbubbles by allowing unshelled bubbles to persist long enough for a lipid monolayer to form on the surface [24]. Further, the increased volume density of Xe-OFP-MB (Fig. 4) is consistent with the observation that Xe-OFP-MB had a

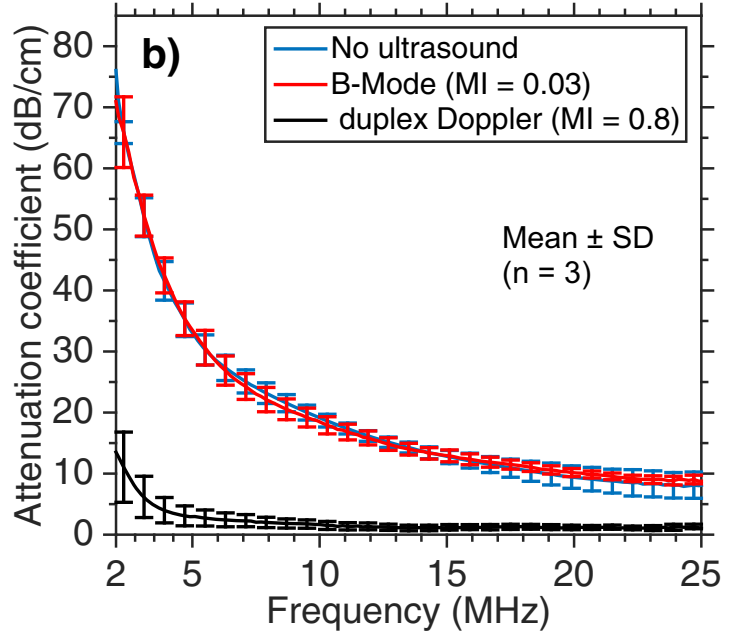

$\mathrm{MB}$ are shown without exposure to ultrasound and after exposure to Bmode $(\mathrm{MI}=0.03)$ and duplex Doppler $(\mathrm{MI}=0.8)$. Note the change in the ordinate scale

higher attenuation coefficient (Fig. 5) and xenon payload (Tables 1 and 2) than Xe-MB.

Xe-OFP-MB were stable in an undersaturated solution $(\mathrm{DO}=87.5 \pm 1.8 \%)$ that caused complete loss of microbubble volume and attenuation in Xe-MB (Figs. 4 and 6). This observation is likely because of the stabilizing effect of OFP [31, 32]. Similar trends were observed previously in studies that focused on oxygen delivery using lipid-shelled microbubbles $[24,30]$. Specifically, co-encapsulation of 5 to $20 \%$ perfluorocarbon gas improved stability markedly relative to microbubbles loaded only with oxygen $[24,30]$.

In vitro imaging (Fig. $7 \mathrm{a}-\mathrm{d}$ ) showed a $24-\mathrm{dB}$ enhancement in echogenicity relative to baseline images for Xe-MB. XeOFP-MB and Definity ${ }^{\circledR}$ showed higher enhancement (29 and $33 \mathrm{~dB}$ ) over the baseline images. The enhancements produced by Definity® and Xe-OFP-MB were similar, consistent with
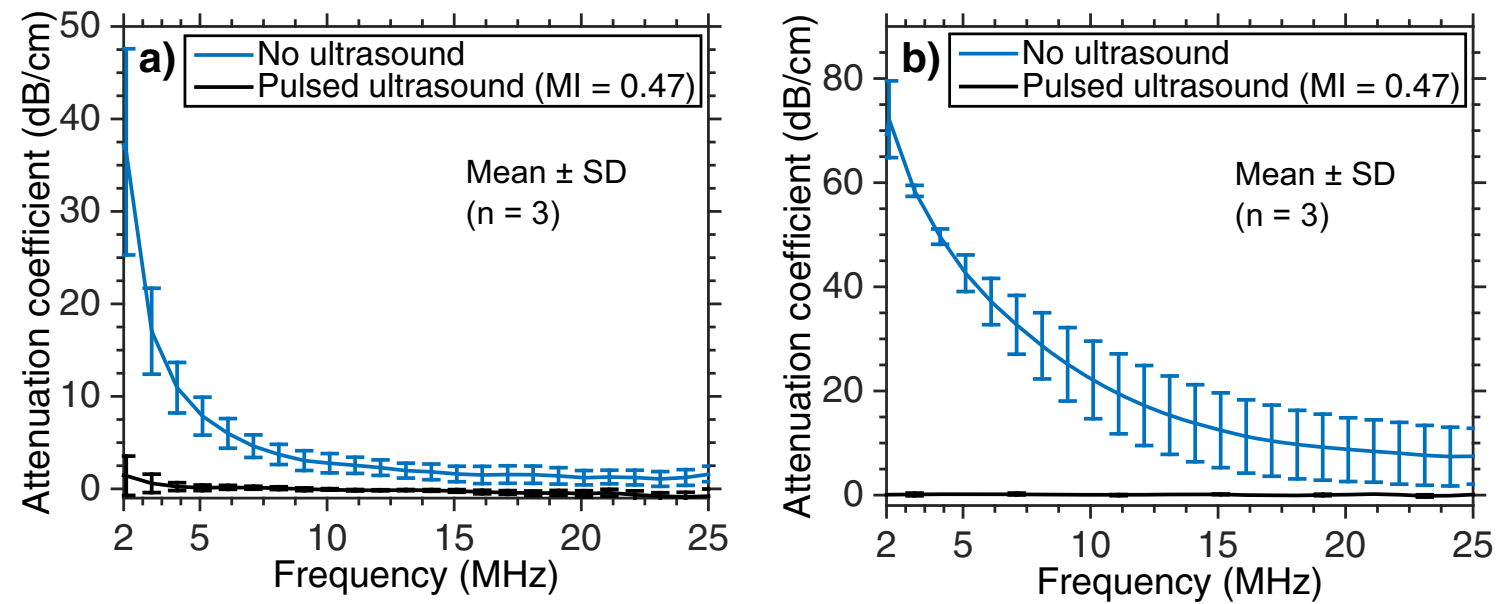

Fig. 10 Frequency-dependent attenuation loss triggered by pulsed ultrasound (220-kHz frequency, 15-cycle pulse length, 5-kHz pulse repetition frequency, MI = 0.47, $10 \mathrm{~s}$ insonation) or sham (no ultrasound) from (a) Xe-MB and (b) Xe-OFP-MB. Note the different ordinate scales of (a) and (b) 
the trend observed from attenuation spectroscopy measurements at $5.2 \mathrm{MHz}$ (Fig. 5). In vivo imaging (Fig. 7e-h) of $\mathrm{Xe}-\mathrm{MB}, \mathrm{Xe}-\mathrm{OFP}-\mathrm{MB}$, and Definity® showed that the contrast produced by Xe-OFP-MB in response to insonation at $18 \mathrm{MHz}$ was higher than Xe-MB and lower than Definity®. At $18 \mathrm{MHz}, \mathrm{Xe-MB}$ had no discernible enhancement, which could be due to the low acoustic responsiveness of this agent at high frequencies, consistent with attenuation spectroscopy measurements (Fig. 5).

The Xe dose in Xe-OFP-MB was higher than Xe-MB. The variability in Xe dose measurements was likely caused by steps such as gas injection into the vial headspace, bubble handling (transfer of sample into a new vial), and loss of gas during injection of small volumes into the GC/MS system. $\mathrm{Xe}-\mathrm{OFP}-\mathrm{MB}$ were more stable than Xe-MB, particularly in an undersaturated solution that mimicked venous gas saturation. The GC/MS studies were performed using undiluted dispersions of Xe-MB and Xe-OFP-MB to achieve adequate signal to noise ratio. Therefore, the fluid containing the microbubbles was saturated with Xe, whereas the fluids used to dilute the microbubble suspensions for attenuation spectroscopy were not Xe-saturated. The total dose of Xe obtained by summing the volume from Coulter counter measurements with published solubility values for Xe in aqueous solution and lipid (Table 1) was in general agreement with GC/MS measurements (Table 2). The GC/MS measurement underestimation may have been due to loss of gas during sample transfer and injection. These observations are consistent with a previous study [38], which reported that GC/MS measurements of Xe-saturated water were able to measure only $64 \%$ of the total Xe dose (volume of gas released into the headspace plus that retained in the Xe-saturated solution).

Microbubbles and echogenic liposomes differ in their Xe release profile and loading capacity. Xe-ELIP have been reported to have a biphasic Xe release profile [17, 19]. Specifically, the Xe payload in gaseous form is released rapidly upon sonication, followed by slow release of lipid dissolved in the lipid bilayer [17]. Xe-ELIP have also been shown to adhere to damaged endothelium, which could improve targeting of Xe to ischemic areas [17]. The total Xe dose in Xe-OFP-MB per milliliter of solution was 1.7-fold lower than in Xe-ELIP $[17,38]$. The lipid concentration per milliliter of solution in Xe-OFP-MB and Xe-ELIP was 1 and $10 \mathrm{mg} / \mathrm{ml}$, after formation (MB) or reconstitution (ELIP), respectively. Because the amount of lipid in Xe-OFP-MB was 10-fold lower than in Xe-ELIP, the total Xe dose in Xe-OFP-MB per milligram of lipid was subsequently 6 -fold higher. Therefore, the suitability of Xe-ELIP or Xe-OFP-MB for in vivo applications might depend upon the desired total amount of lipid injected. Note that the efficacy of Xe-ELIP has been demonstrated extensively in vivo [17-19, 22]. Future preclinical studies are needed to compare the therapeutic efficacy of Xe-loaded microbubbles relative to Xe-ELIP.
Shelf-life studies of Xe-MB and Xe-OFP-MB (Fig. 8) showed a substantial decrease in dose within 28 days of adding $\mathrm{Xe}$ or $\mathrm{Xe} / \mathrm{OFP}$ gas, although the decrease in dose over time was lower for Xe-OFP-MB than Xe-MB. This decrease in dose can be attributed in part due to the leakage of Xe through the septum. The present protocol for loading gas into the vial involves piercing the butyl rubber septum twice- - for evacuating the air, and for adding Xe gas, which could adversely affect gas retention in the vial over time. We expect that the shelf life can be improved further by using industry standard methods of gas loading into the vial.

Attenuation measurements performed after exposure to duplex Doppler ultrasound (Fig. 9) and 220-kHz pulsed ultrasound (Fig. 10) demonstrated a loss of attenuation due to the destruction of the microbubbles. Therefore, sonication could be used to release the Xe gas payload into the surrounding fluid for diffusion into tissues where it could impart neuroprotective effects. Of note, Xe-OFP-MB were stable when insonified with B-mode at a low acoustic output $(\mathrm{MI}=0.03)$. Therefore, this Xe-loaded agent could potentially be used for site-specific delivery of Xe under ultrasound image guidance.

In this study, the measurement of Xe dose was performed under idealized ("in vial") conditions. Furthermore, ultrasound-triggered release was based on attenuation measurements, which served as a surrogate for Xe payload release. Due to the limitations of the GC/MS measurement technique, it could not be confirmed whether the payload released in response to $6-\mathrm{MHz}$ or $220-\mathrm{kHz}$ pulsed ultrasound contained xenon. However, the short time elapsed between agent dilution and payload release with ultrasound $(\sim 20 \mathrm{~s})$ suggests that only a small fraction of the Xe dose would be passively released before ultrasound exposure. This assertion is supported by previous literature on oxygen $[24,30]$ and nitric oxide [58] delivery with similar lipid-shelled microbubbles.

The time taken for the agent to reach the target in vivo will depend on the administration strategy. For the therapy to be successful, majority of the xenon payload should be retained in the microbubbles until ultrasound-triggered release at the target location. In the context of stroke treatment, both intravenous and intra-arterial approaches are clinically relevant [59]. Specifically, apart from intravenous delivery, intraarterial administration of thrombolytic is already in clinical use [60]. In intra-arterial delivery, the microbubbles will reach the target region within a few seconds and the payload can be released rapidly by ultrasound. Either a bolus or a diluted bolus in Xe-saturated saline could be employed for intraarterial approaches. Thus, loss of Xe payload by passive diffusion is not expected to be a challenge for intra-arterial applications. For intravenous applications, the time between in vivo administration and reaching the target location is governed by the blood recirculation time, which is only $15 \mathrm{~s}$ in murine models [49] and nearly $1 \mathrm{~min}$ in humans [61]. Our group has already reported the therapeutic efficacy of Xe- 
ELIP in murine models using both intravenous and intraarterial administration of the agent [17-19, 22]. Moreover, previous studies have shown that co-encapsulation of a perfluorocarbon gas also imparts temporal stability to the bioactive gas through osmotic [32] and co-surfactant effects [31, 57]. Specifically, in vitro studies on oxygen delivery suggest that co-encapsulation of oxygen gas can extend the temporal payload stability $[24,30]$. Therefore, we anticipate that Xeloaded microbubbles will have a therapeutic effect when administered intravenously. Also, the higher dose of Xe-MB and $\mathrm{Xe}-\mathrm{OFP}-\mathrm{MB}$ relative to Xe-saturated and Xe-OFP-saturated solution (Table 2) suggests that these agents will enhance therapeutic efficacy over administration of Xe-saturated saline. However, future large animal and human studies (in which the recirculation time is longer) will be necessary to assess whether intravenous administration of Xe-OFPloaded microbubbles provides neuroprotection during ischemia. Recent reports with microbubbles stabilized with surfactants [28, 62], dextran [63], polymer [64], or chitosan shells [65] show promise for reducing gas diffusion across the shell, even without co-encapsulating perfluorocarbon gases. In the future, the performance and biocompatibility of such agents should be compared against lipid-shelled microbubbles for Xe delivery.

This study had a few notable limitations. The passive release profile of Xe from Xe-OFP-MB could not be assessed in this study because of the limited time resolution of GC/MS measurements. Future studies will assess Xe payload stability using alternate techniques within the first $4 \mathrm{~min}$, consistent with the reported in vivo half-life of a similar lipid-shelled ultrasound contrast agent [66]. The size distributions reported in this study were measured at room temperature. Although the acoustic response of microbubbles varies with temperature $[34,67]$, the size distribution of lipid-shelled contrast agents has been shown to not vary substantially at room and physiological temperatures [37]. Future in vivo studies will be necessary to assess the therapeutic efficacy of Xe delivery using the agents reported in this study.

\section{Conclusions}

The findings of this study demonstrate that Xe can be loaded within lipid-shelled microbubbles for ultrasound-triggered release. Co-encapsulation of octafluoropropane gas enhanced the Xe dose, volume, and stability of Xe-OFP-MB. Xe-MB and Xe-OFP-MB encapsulated $113.8 \pm 16.1$ and $145.6 \pm$ $25.5 \mu \mathrm{l}$ of Xe per $\mathrm{mg}$ of lipid, respectively. The feasibility of gas payload release was demonstrated with 6-MHz duplex Doppler and $220-\mathrm{kHz}$ pulsed ultrasound. These results suggest that lipid-shelled microbubbles could serve as ultrasoundtriggered xenon delivery agents for applications such as neuroprotection in stroke.
Acknowledgments This work was supported by the National Institutes of Health/National Institute of Neurological Disorders and Stroke through grant R01 NS047603. Kevin Haworth, Ph.D., was supported by the National Institutes of Health/National Heart, Lung, and Blood Institute through grant K25HL133452. The authors thank Prof. Jack Rubinstein, Dr. Sheryl Koch, and Michelle Nieman for their help with in vivo imaging and Dr. Karla Mercado-Shekhar for assistance with in vitro imaging. Prof. Dong Zhang and Prof. Xiasheng Guo are acknowledged for providing the $220-\mathrm{kHz}$ transducer used in this study, and Robert Kleven for calibrating it. The authors are grateful to Prof. Kenneth Setchell for sharing his expertise on gas chromatography/mass spectroscopy measurements.

Required Author Forms Disclosure forms provided by the authors are available with the online version of this article.

\section{References}

1. Benjamin EJ, Virani SS, Callaway CW, et al. Heart disease and stroke statistics - 2018 update: a report from the American Heart Association. Circulation. 2018;137:e67-e492.

2. Majid A. Neuroprotection in stroke: past, present, and future. ISRN Neurol 2014;2014:515716.

3. Moskowitz MA, Lo EH, Iadecola C. The science of stroke: mechanisms in search of treatments. Neuron. 2010;67:181-198.

4. Hwang BY, Appelboom G, Ayer A, et al. Advances in neuroprotective strategies: potential therapies for intracerebral hemorrhage. Cerebrovasc Dis 2011;31:211-222.

5. Laskowitz DT, Kolls BJ. Neuroprotection in subarachnoid hemorrhage. Stroke. 2010;41:S79-S84.

6. Lai TW, Zhang S, Wang YT. Excitotoxicity and stroke: identifying novel targets for neuroprotection. Prog Neurobiol 2014;115:157188.

7. Rajah GB, Ding Y. Experimental neuroprotection in ischemic stroke: a concise review. Neurosurg Focus 2017;42:E2.

8. Fan X, Kavelaars A, Heijnen CJ, Groenendaal F, van Bel F. Pharmacological neuroprotection after perinatal hypoxic-ischemic brain injury. Curr Neuropharmacol 2010;8:324-334.

9. Fisher M. New approaches to neuroprotective drug development. Stroke. 2011;42:S24-S27.

10. Preckel B, Weber NC, Sanders RD, Maze M, Schlack W. Molecular mechanisms transducing the anesthetic, analgesic, and organprotective actions of xenon. Anesthesiology. 2006;105:187-197.

11. Sanders RD, Ma D, Maze M. Anaesthesia induced neuroprotection. Best Pract Res Clin Anaesthesiol 2005;19:461-474.

12. Dickinson R, Franks NP. Bench-to-bedside review: molecular pharmacology and clinical use of inert gases in anesthesia and neuroprotection. Crit Care 2010;14.

13. Ma D, Hossain M, Pettet GK, et al. Xenon preconditioning reduces brain damage from neonatal asphyxia in rats. J Cereb Blood Flow Metab 2006;26:199-208.

14. Koerner IP, Brambrink AM. Brain protection by anesthetic agents. Curr Opin Anaesthesiol 2006;19:481-486.

15. Adibhatla RM, Hatcher JF. Tissue plasminogen activator (tPA) and matrix metalloproteinases in the pathogenesis of stroke: therapeutic strategies. CNS Neurol Disord Drug Targets 2008;7:243-253.

16. Dingley J, Findlay GP, Foex BA, et al. A closed xenon anesthesia delivery system. Anesthesiology. 2001;94:173-176.

17. Miao YF, Peng T, Moody MR, et al. Delivery of xenon-containing echogenic liposomes inhibits early brain injury following subarachnoid hemorrhage. Sci Rep 2018;8:450. 
18. Peng T, Britton GL, Kim H, et al. Therapeutic time window and dose dependence of xenon delivered via echogenic liposomes for neuroprotection in stroke. CNS Neurosci Ther 2013;19:773-784.

19. Britton GL, Kim H, Kee PH, et al. In vivo therapeutic gas delivery for neuroprotection with echogenic liposomes. Circulation. 2010;122:1578-1587.

20. Kim H, Britton GL, Peng T, et al. Nitric oxide-loaded echogenic liposomes for treatment of vasospasm following subarachnoid hemorrhage. Int J Nanomedicine 2014;9:155-165.

21. Huang SL, Kee PH, Kim H, et al. Nitric oxide-loaded echogenic liposomes for nitric oxide delivery and inhibition of intimal hyperplasia. J Am Coll Cardiol 2009;54:652-659.

22. Dandekar MP, Peng T, McPherson DD, et al. Intravenous infusion of xenon-containing liposomes generates rapid antidepressant-like effects. Prog Neuro-Psychopharmacol Biol Psychiatry 2018;86: 140-149.

23. Huang SL, McPherson DD, Macdonald RC. A method to coencapsulate gas and drugs in liposomes for ultrasound-controlled drug delivery. Ultrasound Med Biol 2008;34:1272-1280.

24. Kwan JJ, Kaya M, Borden MA, Dayton PA. Theranostic oxygen delivery using ultrasound and microbubbles. Theranostics. 2012;2: 1174-1184.

25. Wang $\mathrm{C}$, Yang $\mathrm{F}, \mathrm{Xu} \mathrm{ZH}$, et al. Intravenous release of $\mathrm{NO}$ from lipidic microbubbles accelerates deep vein thrombosis resolution in a rat model. Thromb Res 2013;131:E31-E38.

26. Fix SM, Borden MA, Dayton PA. Therapeutic gas delivery via microbubbles and liposomes. J Control Release 2015;209:139149.

27. Chen G, Yang L, Zhong L, et al. Delivery of hydrogen sulfide by ultrasound targeted microbubble destruction attenuates myocardial ischemia-reperfusion injury. Sci Rep 2016;6:30643.

28. Eisenbrey JR, Shraim R, Liu JB, et al. Sensitization of hypoxic tumors to radiation therapy using ultrasound-sensitive oxygen microbubbles. Int J Radiat Oncol 2018;101:88-96.

29. Owen J, McEwan C, Nesbitt H, et al. Reducing tumour hypoxia via oral administration of oxygen nanobubbles. PLoS One 2016;11.

30. Yang CJ, Xiao H, Sun Y, et al. Lipid microbubbles as ultrasoundstimulated oxygen carriers for controllable oxygen release for tumor reoxygenation. Ultrasound Med Biol 2018;44:416-425.

31. Szijjarto C, Rossi S, Waton G, Krafft MP. Effects of perfluorocarbon gases on the size and stability characteristics of phospholipid-coated microbubbles: osmotic effect versus interfacial film stabilization. Langmuir. 2012;28:1182-1189.

32. Kabalnov A, Bradley J, Flaim S, et al. Dissolution of multicomponent microbubbles in the bloodstream: 2. Experiment. Ultrasound Med Biol 1998;24:751-760.

33. Goertz DE, de Jong N, van der Steen AFW. Attenuation and size distribution measurements of definity (TM) and manipulated definity (TM) populations. Ultrasound Med Biol 2007;33:13761388.

34. Raymond JL, Haworth KJ, Bader KB, et al. Broadband attenuation measurements of phospholipid-shelled ultrasound contrast agents. Ultrasound Med Biol 2014:40:410-421.

35. Altman PL. Handbook of respiration. Philadelphia: W. B. Saunders; 1959.

36. Mulvana H, Stride E, Tang MX, Hajnal JV, Eckersley RJ. The influence of gas saturation on microbubble stability. Ultrasound Med Biol 2012;38:1097-1100.

37. Shekhar H, Smith NJ, Raymond JL, Holland CK. Effect of temperature on the size distribution, shell properties, and stability of definity (R). Ultrasound Med Biol 2018;44:434-446.

38. Klegerman ME, Moody MR, Hurling JR, et al. Gas chromatography/mass spectrometry measurement of xenon in gas-loaded liposomes for neuroprotective applications. Rapid Commun Mass Spectrom 2017;31:1-8.
39. Yeh SY, Peterson RE. Solubility of carbon dioxide, krypton, and xenon in lipids. J Pharm Sci 1963;52:453-458.

40. Yeh SY, Peterson RE. Solubility of carbon dioxide, krypton, and xenon in aqueous solution. J Pharm Sci 1964;53:822-824.

41. Tetreau C, Blouquit Y, Novikov E, Quiniou E, Lavalette D. Competition with xenon elicits ligand migration and escape pathways in myoglobin. Biophys J 2004;86:435-447.

42. King AD. The solubility of gases in aqueous solutions of poly(propylene glycol). J Colloid Interface Sci 2001;243:457-462.

43. Goto T, Suwa K, Uezono S, et al. The blood-gas partition coefficient of xenon may be lower than generally accepted. Br J Anaesth 1998;80:255-256.

44. Kenwright DA, Thomson AJ, Hadoke PW, et al. A protocol for improved measurement of arterial flow rate in preclinical ultrasound. Ultrasound Int Open 2015;1:E46-52.

45. Kubo-Inoue M, Egashira K, Usui M, et al. Long-term inhibition of nitric oxide synthesis increases arterial thrombogenecity in rat carotid artery. Am J Physiol-Heart C 2002;282:H1478-H1484.

46. McDannold N, Arvanitis CD, Vykhodtseva N, Livingstone MS. Temporary disruption of the blood-brain barrier by use of ultrasound and microbubbles: safety and efficacy evaluation in rhesus macaques. Cancer Res 2012;72:3652-3663.

47. Verbree J, Bronzwaer ASGT, Ghariq E, et al. Assessment of middle cerebral artery diameter during hypocapnia and hypercapnia in humans using ultra-high-field MRI. J Appl Physiol 2014;117: 1084-1089.

48. Alexandrov AV, Tsivgoulis G, Rubiera M, et al. End-diastolic velocity increase predicts recanalization and neurological improvement in patients with ischemic stroke with proximal arterial occlusions receiving reperfusion therapies. Stroke. 2010;41:948-952.

49. Debbage PL, Griebel J, Ried M, et al. Lectin intravital perfusion studies in tumor-bearing mice: micrometer-resolution, wide-area mapping of microvascular labeling, distinguishing efficiently and inefficiently perfused microregions in the tumor. J Histochem Cytochem 1998;46:627-639.

50. Widmaier EP, Raff H, Strang KT, Vander AJ. Vander's human physiology: the mechanisms of body function. Fourteenth edition. ed. New York, NY: McGraw-Hill; 2016. 1 volume (various pagings) $\mathrm{p}$.

51. Borden MA, Longo ML. Dissolution behavior of lipid monolayercoated, air-filled microbubbles: effect of lipid hydrophobic chain length. Langmuir. 2002;18:9225-9233.

52. Garg S, Thomas AA, Borden MA. The effect of lipid monolayer inplane rigidity on in vivo microbubble circulation persistence. Biomaterials. 2013;34:6862-6870.

53. Kim DH, Costello MJ, Duncan PB, Needham D. Mechanical properties and microstructure of polycrystalline phospholipid monolayer shells: novel solid microparticles. Langmuir. 2003;19:84558466.

54. Borden MA, Longo ML. Oxygen permeability of fully condensed lipid monolayers. J Phys Chem B 2004;108:6009-6016.

55. Chen CC, Borden MA. Ligand conjugation to bimodal poly(ethylene glycol) brush layers on microbubbles. Langmuir. 2010;26: 13183-13194.

56. Sarkar K, Katiyar A, Jain P. Growth and dissolution of an encapsulated contrast microbubble: effects of encapsulation permeability. Ultrasound Med Biol 2009;35:1385-1396.

57. Abou-Saleh RH, Peyman SA, Johnson BRG, et al. The influence of intercalating perfluorohexane into lipid shells on nano and microbubble stability. Soft Matter 2016;12:7223-7230.

58. Sutton JT, Raymond JL, Verleye MC, Pyne-Geithman GJ, Holland CK. Pulsed ultrasound enhances the delivery of nitric oxide from bubble liposomes to ex vivo porcine carotid tissue. Int $\mathrm{J}$ Nanomedicine 2014;9:4671-4683. 
59. Cloft HJ, Rabinstein A, Lanzino G, Kallmes DF. Intra-arterial stroke therapy: an assessment of demand and available work force. Am J Neuroradiol 2009;30:453-458.

60. Abou-Chebl A. Intra-arterial therapy for acute ischemic stroke. Neurotherapeutics. 2011;8:400-413.

61. Widmaier EP, Raff H, Strang KT. Vander's human physiology: the mechanisms of body function. 2006.

62. Eisenbrey JR, Albala L, Kramer MR, et al. Development of an ultrasound sensitive oxygen carrier for oxygen delivery to hypoxic tissue. Int J Pharm 2015;478:361-367.

63. Cavalli R, Bisazza A, Giustetto P, et al. Preparation and characterization of dextran nanobubbles for oxygen delivery. Int $\mathrm{J}$ Pharm 2009;381:160-165.

64. McEwan C, Fowley C, Nomikou N, et al. Polymeric microbubbles as delivery vehicles for sensitizers in sonodynamic therapy. Langmuir. 2014;30:14926-14930.
65. Cavalli R, Bisazza A, Rolfo A, et al. Ultrasound-mediated oxygen delivery from chitosan nanobubbles. Int J Pharm 2009;378:215217.

66. Fix SM, Nyankima AG, McSweeney MD, et al. Accelerated clearance of ultrasound contrast agents containing polyethylene glycol is associated with the generation of anti-polyethylene glycol antibodies. Ultrasound Med Biol 2018;44:1266-1280.

67. Mulvana H, Stride E, Tang M, Hajnal JV, Eckersley R. Temperature-dependent differences in the nonlinear acoustic behavior of ultrasound contrast agents revealed by high-speed imaging and bulk acoustics. Ultrasound Med Biol 2011;37:1509-1517.

Publisher's Note Springer Nature remains neutral with regard to jurisdictional claims in published maps and institutional affiliations. 